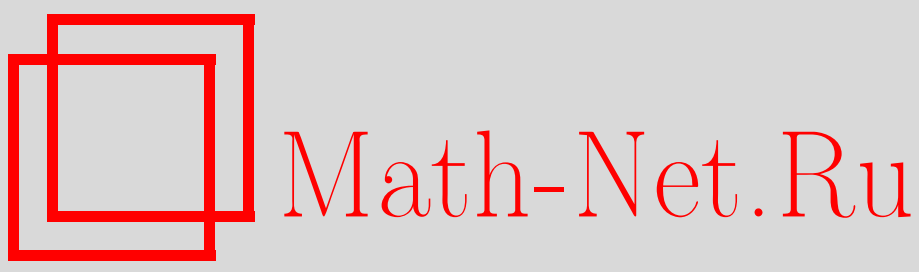

А. И. Никонов, Критериально-множественная основа формирования операционных моделей физических цепей, Вестн. Сам. гос. техн. ун-та. Сер. Физ.-мат. науки, 2004, выпуск 26, 174-179

DOI: https://doi.org/10.14498/vsgtu195

Использование Общероссийского математического портала Math-Net.Ru подразумевает, что вы прочитали и согласны с пользовательским соглашением

http://www. mathnet.ru/rus/agreement

Параметры загрузки:

IP : 52.6 .47 .48

26 апреля 2023 г., 15:10:00

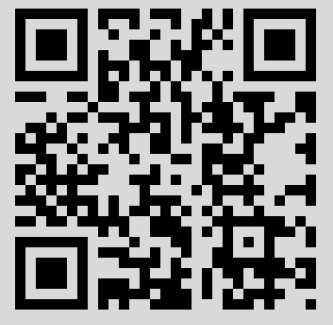




\section{КРИТЕРИАЛЬНО-МНОЖЕСТВЕННАЯ ОСНОВА ФОРМИРОВАНИЯ ОПЕРАЦИОННЫХ МОДЕЛЕЙ ФИЗИЧЕСКИХ ЦЕПЕЙ}

Приведены количественные критерии подобия физических ичепей, содержащче величины- аналоги, а также параметры- аналоги. Выявлены вещзественно- числовые и вещуественно- функциональные множества, элементы которых могут выбираться для операционного моделирования физических uุeneй.

Сходство, присущее количественным описаниям процессов в электрической и механической цепях, подвергаемых воздействиям соответственно электрического напряжения и механической силы, хорошо известно; известен также подход к моделированию физических цепей, согласно которому каждой из определенного набора величин какой-либо физической природы ставится в соответствие определенная величина- аналог другой природы $[1,2]$. Для электрической, магнитной, механической, гидравлической цепей эти аналоги могут быть выражены, в частности, соответствиями “электрический заряд - потокосцепление -перемещение - объем". Присвоим указанным цепям индексы соответственно э, $\mu, \mu, h$.

В любой физической цепи, поддающейся моделированию данного вида, принято выделять типовые параметрические элементы, которые, пользуясь электротехнической терминологией, называют емкостным, резистивным, индуктивным. Их параметры-аналоги обозначим соответственно через $C_{a}, R_{a}, L_{a}$, причем $a \in\{\ni, \mu, M, h\}$; кроме того, введем обозначение обобщенного параметра типового элемента физической цепи:

$$
\Pi_{a} \in\left\{C_{a}, R_{a}, L_{a}\right\} .
$$

Выражения взаимосвязей величин- аналогов электрического заряда $(q)$, электрического напряжения $(u)$, тока $(i)$, потокосцепления $(\psi)$ в количественном описании цепи произвольной природы представляют собой критерии подобия физических цепей [2], например, критерии дифференцирования заряда и действия резистивного элемента имеют вид

$$
i=d q / d t, \quad u=R_{a} i,
$$

где $t$ - время. Величины $q, i, u, \psi$ можно рассматривать как входы и выходы элементов, выполняющих определенные операции, и эти элементы можно графически отобразить в соответствующих операционных схемах.

Построение моделей-аналогов физических цепей, производимое на основе критериев подобия и операционных структур, обеспечивает возможность выполнения укрупнений и детализаций моделируемых системных блоков, в том числе, переходов к их подробному принципиально-схемному представлению. Предусматривая количественное отображение принципа действия и характеристик моделируемого объекта, рассматриваемый подход обеспечивает прослеживаемость распространения и преобразования входных воздействий на данный объект, однозначность восприятия действия как объекта в целом, так и его составных частей.

Однако формирование операционных моделей-аналогов физических цепей до настоящего времени не обосновывалось каким-либо математическим оформлением числовых, функциональных структур, служащих диапазонной основой операционных аналогий. Отсутствие же сведений, касающихся указанной основы, может приводить к возникновению существенной неадекватности операционных описаний, что, в свою очередь, может отрицательно сказываться на качестве разработки, контроля, совершенствования моделируемых объектов. Цель настоящей работы состоит в выявлении вещественно-числовых и вещественно-функциональных множеств, элементы которых выбираются при моделировании физических цепей для значимого воспроизведения критериев подобия, применяемых для построения операционно-модельных схем с учетом их параметрической специфики.

Применительно к модели произвольной физической цепи взаимосвязь величин-аналогов в критериях подобия осуществляется посредствам операций умножения (со множителями типа $\left.\Pi_{a}\right)$ :

$$
q=q_{c}=C_{a} u_{c}, u=u_{R}=R_{a} i_{R}, \psi=\psi_{L}=L_{a} i_{L} .
$$

Здесь $q_{c}, u_{c}$ - величины-аналоги электрических заряда и напряжения, выделяемые в емкостном элементе; $u_{R}, i_{R}$ - величины-аналоги электрических напряжений и тока, выделяемые в резистив-

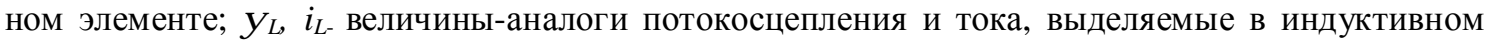


элементе. Итак, обозначения величин $q, i, u, \psi$ снабжены индексами, указывающими на их принадлежность моделям тех или иных параметрических элементов.

Схемно-операционное представление критериев (1), выполняемое звеньями емкостного, резистивного и индуктивного типов, иллюстрирует рис. 1. Кроме того, к критериям подобия величин физических цепей относятся выражения временного дифференцирования и интегрирования вида

$$
\begin{gathered}
i=q^{\prime}=d q / d t ; \quad i^{\prime}=d i / d t=d^{2} q / d t^{2} ; u^{\prime}=d u / d t ; \\
q=\int_{I_{t}} i d t ; \quad i=\int_{I_{t}} i^{\prime} d t ; \quad u=\int_{I_{t}} u^{\prime} d t ; \quad \psi=\int_{I_{t}} u d t,
\end{gathered}
$$

где $I_{t}$ - конечный интервал действия, наблюдения величин-аналогов; начальный момент интервала $I_{t}$ обозначим через $t_{0}$.

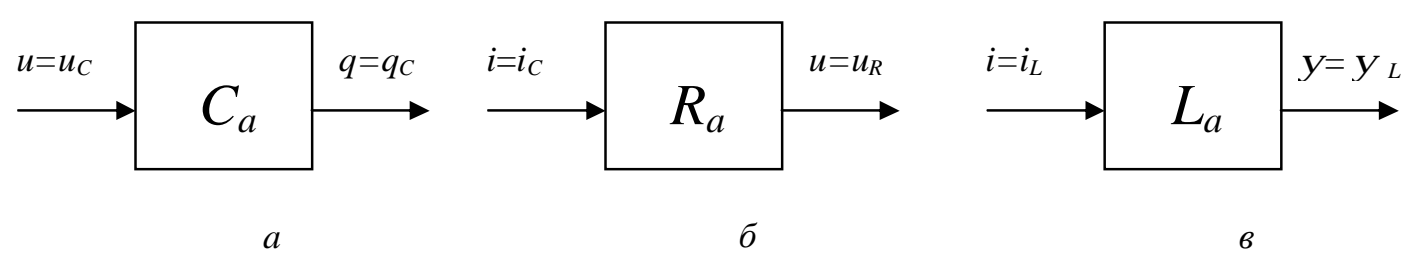

Рис. 1. Схемно-операционное изображение емкостного (a), резистивного (б), индуктивного (в) параметрических звеньев

На рис. 2 изображены схемно-операционные модельные звенья дифференцирования $(a)$ и интегрирования (б) обобщенной величины $b_{b x} \in\{q, i, u\}$. Полагается также, что выходная величина звена $B_{\text {gыx }} \in\left\{q, i, u, u^{\prime}, \Psi\right\} ; k_{d i f}=d(\cdot) / d t$ - операторный коэффициент дифференцирования; $k_{s}=\int_{I_{t}}(\cdot) d t$ - операторный коэффициент интегрирования; позиция вида (·) предназначена для размещения символа величины, играющей роль входной применительно к данной операции.

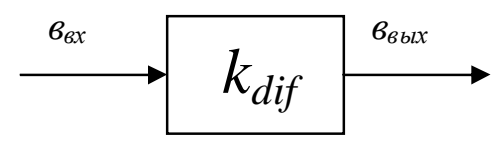

$a$

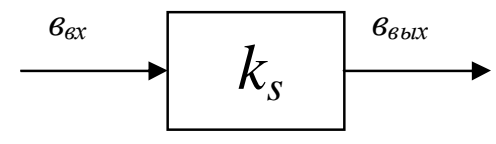

6

Р ис.2. Схемно-операционное изображение дифференцирующего (a) и интегрирующего (б) звеньев

Из выражений (1)-(3) нетрудно получить следующие соотношения:

$$
\begin{gathered}
i=i_{c}=d\left(C_{a} u_{C}\right) / d t=C_{a} u_{C}^{\prime}+C_{a}^{\prime} u_{C} ; \\
u=u_{L}=d\left(L_{a} i_{L}\right) / d t=L_{a} i_{L}^{\prime}+L_{a}^{\prime} i_{L} ; \\
u_{C}^{\prime}=d u_{C} / d t, \quad C_{a}^{\prime}=d C_{a} / d t, \quad i_{L}^{\prime}=d i_{L} / d t, \quad L_{a}^{\prime}=d L_{a} / d t .
\end{gathered}
$$

Допущение о постоянстве или квазипостоянстве параметров типа $\Pi_{a}^{\prime}=d \Pi_{a} / d t$ позволяет привести соотношения (4)-(5) к частному виду $i_{C}=C_{a} u_{C}^{\prime}, u_{L}=L_{a} i_{L}^{\prime}$.

Из критериальных выражений (1)-(5) следует, что входящие в них величины $i, i^{\prime}, u^{\prime}, \psi$ получаются путем временного дифференцирования и временного интегрирования аналогов заряда и напряжения $(q, u)$, которые имеет смысл именовать образующими величинами - аналогами. Образующие и производные величины аналоги выдаются источниками, способными реально развивать конечную мощность, поэтому и сами они могут считаться конечными.

Свойства величин - аналогов, связанных с описанием действия индуктивного и емкостного параметрических параметров, согласуются с законами коммутации [3], сфера приложения сущ- 
ности которых выходит за электротехнические рамки и имеет более общий характер. Согласно таким обобщенным законам ни ток - аналог индуктивного элемента $i_{L}$, ни напряжение - аналог емкостного элемента $u_{C}$ не могут изменяться скачкообразно.

Нормированная форма критериев подобия величин, относящихся к цепям различной природы, получается путем деления компонентов соотношений (1) - (5) на соответствующие физические единицы. Итак, критерии подобия могут быть изоморфно представлены:

1) для емкостного параметрического элемента - соотношениями

$$
v_{q C}=v_{C} v_{u C}, \quad v_{i C}=v_{C}^{\prime} v_{u C}+v_{C} v_{u C}^{\prime} ; \quad v_{C}^{\prime}=0 \Rightarrow v_{i C}=v_{C} v_{u C}^{\prime} ;
$$

2) для резистивного элемента - соотношением

$$
v_{u R}=v_{R} v_{i R}
$$

3) для индуктивного элемента - соотношениями

$$
v_{\psi L}=v_{L} v_{i L}, \quad v_{u L}=v_{L}^{\prime} v_{i L}+v_{L} v_{i L}^{\prime} ; \quad v_{L}^{\prime}=0 \Rightarrow v_{u L}=v_{L} v_{i L}^{\prime} .
$$

Здесь форма любой величины типа $v_{\theta \Pi} \in\left\{v_{q C}, v_{u C}, v_{i C}, v_{u C}^{\prime}, v_{u R}, v_{i R}, v_{\psi L}, v_{i L}, v_{u L}, v_{i L}^{\prime}\right\}$ и любого параметра, относящегося к типам $v_{\text {п }} \in\left\{v_{C}, v_{R}, v_{L}\right\}, v_{\Pi} \in\left\{v_{C}^{\prime}, v_{L}^{\prime}\right\}$, безразмерна, поскольку

$$
\begin{aligned}
& v_{6 \Pi}=\frac{B_{\Pi}}{l\left[\varepsilon_{\Pi}\right]}, v_{\Pi}=\frac{\Pi_{a}}{l\left[\Pi_{a}\right]}, v_{6 \Pi}^{\prime}=\frac{B_{\Pi}^{\prime}}{l\left[B_{\Pi}^{\prime}\right]}, v_{\Pi}^{\prime}=\frac{\Pi_{a}^{\prime}}{l\left[\Pi_{a}^{\prime}\right]} ; \\
& \beta_{\Pi} \in\left\{q_{C}, u_{C}, i_{C}, u_{R}, i_{R}, \psi_{L}, i_{L}, u_{L}\right\}, в_{\Pi}^{\prime} \in\left\{u_{C}^{\prime}, i_{L}^{\prime}\right\} \text {. }
\end{aligned}
$$

Выражения для величин $v_{i C}$ и $v_{u L}$ взаимно симметричны по парам индексов $C$ - $L, u$ - $i$; совокупная замена указанных индексов в одном из них (на парные индексы) позволяет сразу получить другое.

Безразмерные критериальные величины, входящие в соотношения (6) - (8), оказывается возможным распределить по множествам, свойства которых отвечают требованиям конечности. Такое распределение отображает следующие включения принадлежности (рис. 3):

$$
\begin{gathered}
v_{u C} \in M_{C 1} I_{t} ; \\
v_{q C}, v_{u C}^{\prime}, v_{i L}, v_{\psi L} \in M_{C 0} I_{t} ; \\
v_{i C}, v_{u R}, v_{i R}, v_{i L}^{\prime}, v_{u L} \in M_{Q} I_{t} ; \\
M_{C l} I_{t} \subset M_{C 0} I_{t} \subset M_{Q} I_{t}=M_{v \theta} I_{t}
\end{gathered}
$$

где $M_{C l} I_{t}, M_{C O} I_{t}, M_{Q} I_{t}$ - множества элементов из пространств соответственно непрерывно дифференцируемых функций $\left(C_{l}\left[t_{0}, t\right]\right)$, непрерывных функций $\left(C_{0}\left[t_{0}, t\right]\right)$, кусочно - непрерывных функций $\left(Q\left[t_{0}, t\right]\right)[4]$. Полагая сначала параметры типа $v_{\text {п }}\left(\Pi_{a}\right)$ постоянными либо квазипостоянными, отнесем их значения к рассмотренной прямой $\bar{R}[5,6]$.

Входы - выходы множества $M_{v 6} I_{t}=M_{Q} I_{t}$ (рис. 3) связаны с типами выбираемых из него величин согласно характеру включений (9) - (11); $O Y_{j}(j=1, \ldots, 5)$ - блок операций умножения парных величин, поступающих на его входы. Типы входных величин блоков умножения составляют множество $\Gamma_{\text {вхоу }}=\left\{v_{u C}, v_{u C}^{\prime}, v_{i L}, v_{i R}, v_{i L}^{\prime}\right\}$, а типы выходных величин данных блоков множество $\Gamma_{\text {выхоу }}=\left\{v_{q C}, v_{i C}, v_{u R}, v_{\psi L}, \psi_{u L}\right\}$.

В случае принятия каким-либо параметром $v_{\text {п }}$ бесконечно большого значения $\left(v_{\Pi}= \pm \infty\right)$ при любом конечном выходе $b_{\text {выхоуj }} \in \Gamma_{\text {выхоу }}$, который он обязан иметь согласно заданным выше исходным требованиям, конечная входная величина данного блока умножения $b_{\text {вxоуj }} \in \Gamma_{\text {вхоу }}$ не может принимать никаких значений, кроме нулевого.

Схема связи исходных множеств критериальных величин и переменных параметров типа $\mathrm{V}_{n}(t)$, относящихся к математическому выражению критериев подобия, приведена на рис. 4. Воспроизведение этих критериев предусматривает использование блоков как операций умно- 
жения $(0 У 1, \ldots, 0 У 7)$, так и операций суммирования $(\Sigma 1, \Sigma 2)$, при этом учитывается влияние производных $C_{a}^{\prime}, L_{a}^{\prime}$ (в безразмерной форме - $\left.v_{C}^{\prime}, v_{L}^{\prime}\right)$.

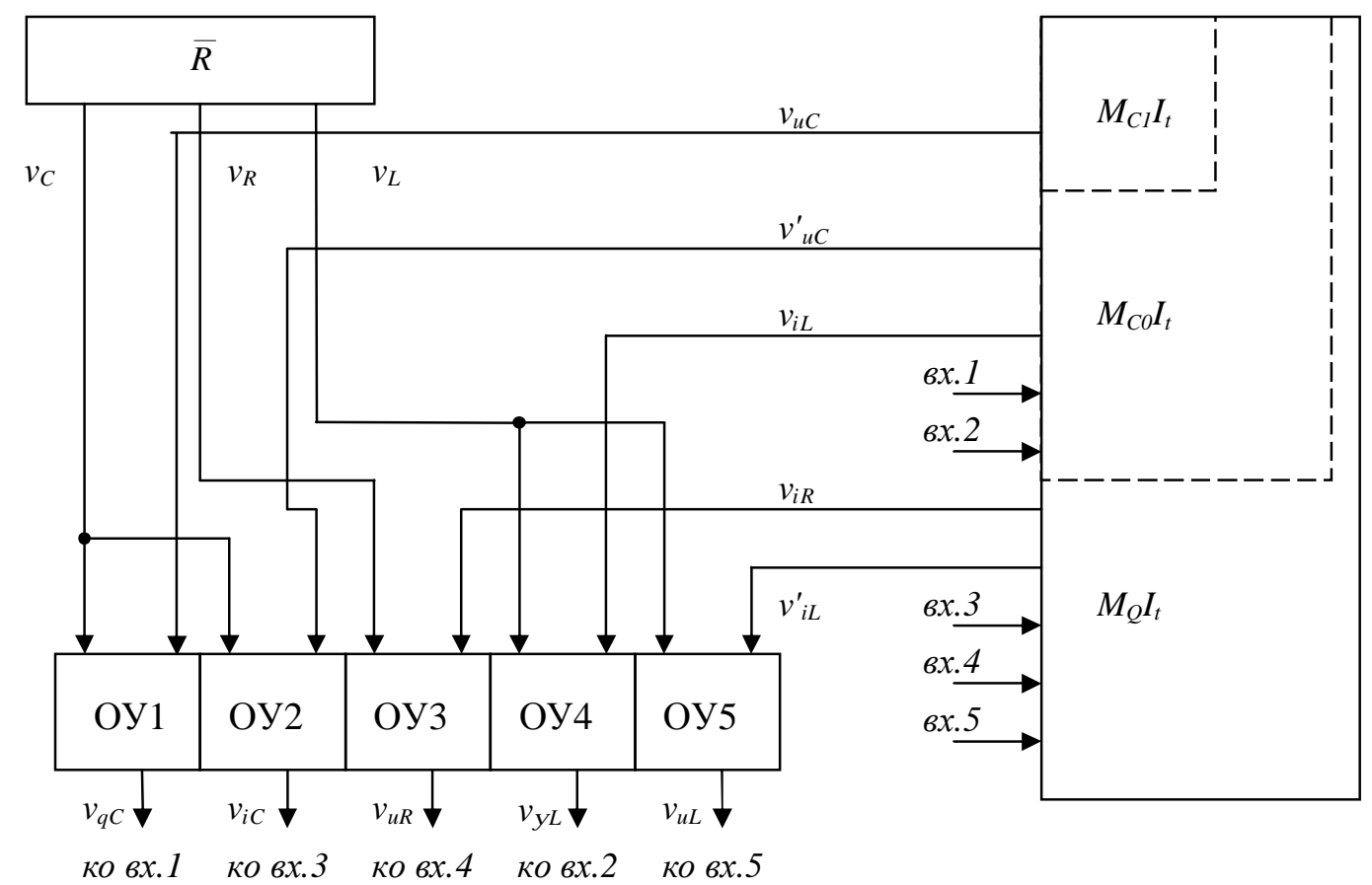

Р ис. 3. Взаимосвязи критериев подобия со множествами критериальных величин и постоянных параметров

Множество входных величин блоков умножения ( $\left.\bar{\Gamma}_{\text {вхоу }}\right)$ остается здесь тем же, что и в предыдущем варианте связи критериев подобия и множествами реализации используемых величин и параметров, т.е. $\bar{\Gamma}_{\text {вхоу }}=\Gamma_{\text {вхоу }}$, а все возможные величины типов

$$
v_{C l}=v_{u C}, v_{C 0} \in\left\{v_{q C}, v_{u C}^{\prime}, v_{i L}, v_{\psi L}\right\} \cup\left\{v_{u C}\right\}, v_{Q} \in\left\{v_{i C}, v_{u R}, v_{i R}, v_{i L}^{\prime}, v_{u L}\right\} \cup\left\{v_{C 0}\right\}
$$

таким же образом, что и прежде, составляют множество $M_{v b} I_{t}$, являющееся множеством кусочно-непрерывных функций $M_{Q} I_{t}$ с подмножествами $M_{C I} I_{t}, M_{C O} I_{t}$. Множество используемых здесь переменных параметров $M_{v п} I_{t}$ представляет собой объединение множества $M_{Q} I_{t}$ с бесконечно большими значениями, принимаемыми параметрами типа $v_{\text {п }}$ на множестве локальных подинтервалов (типа $\Delta t$ ) интервала $I_{t}$ :

$$
M_{v \Pi} I_{t}=M_{Q} I_{t} \cup\left\{ \pm\left.\infty\right|_{\Delta_{t}}, \Delta_{t}=\left[\tau_{0}, t>\tau_{0} \geq t_{0}\right]\right\} ; \tau_{0}, t \in I_{t} .
$$

Дифференцирование временных зависимостей конечных уровней из множества $M_{v п} I_{t}$ дает функции, вновь относящиеся к данному множеству, что относится и к результатам дифференцирования, имеющим бесконечные уровни. В каждом сечении множества $M_{v \pi} I_{t}$, приходящегося на произвольный момент $t=\tau \in I_{t}$, набор получаемых при этом значений $\left.M_{v I} I_{t}\right|_{\tau}$ является ни чем иным, как расширенной прямой $\bar{R}$. Критерии подобия и множества их компонентов, соответствующие постоянству значений модельных параметров, представляют собой, таким образом, частный случай рассматриваемого критериально-множественного варианта, способного учитывать наличие производных $v_{\mathrm{C}}^{\prime}, v_{\mathrm{L}}^{\prime}$ (рис. 4). 


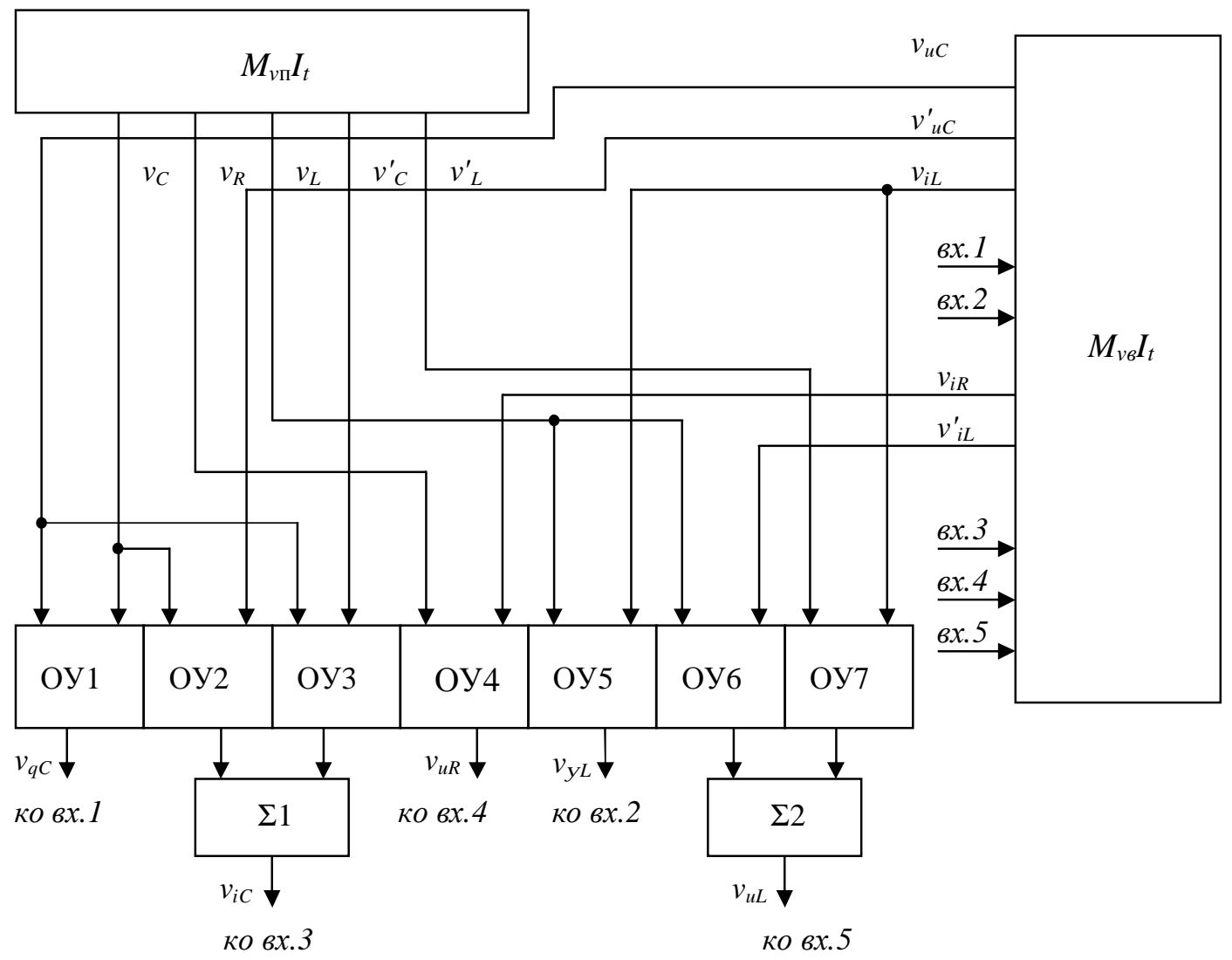

Рис. 4. Взаимосвязи критериев подобия со множествами критериальных величин и переменных параметров

Переход к множествам с размерными величинами $\left(M_{\theta} I_{t}\right)$ и параметрами $\left(M_{\mathrm{n}} I_{t}\right)$, изоморфным и по отношению к $M_{v g} I_{t}$ и $M_{v \pi} I_{t}$, выполняется путем указания физических размерностей величин из $M_{b} I_{t}$, параметров из $M_{\text {п }} I_{t}$, т.е. с помощью умножения элементов безразмерного множества на соответствующую физическую единицу. При действии в каком-либо соединении модельных параметрических звеньев единой величины-аналога её реализации выбираются из того допустимого множества, которое накладывает на нее большие ограничения, и здесь учитывается, что в порядке ужесточения требований к дифференцируемости своих элементов следуют множества $M_{Q} I_{t}, M_{C 0} I_{t}, M_{C l} I_{t}$. Так, применительно к ситуации прохождения токааналога $i$ через последовательное соединение индуктивного и резистивного элементов, к которому приложено напряжение-аналог $u$, в случае $L_{a}=$ const действительны следующие соотношения:

$$
L_{a} i^{\prime}+R_{a} i=u ; \quad L_{a} i^{\prime}=u_{L}, \quad R_{a} i=u_{R}, \quad i=i_{L}=i_{R} .
$$

Согласно схеме взаимосвязей множеств величин и параметров с критериями подобия (составленной с учетом требований законов коммутации) для величин $i_{L}, i_{R}$, выражаемых в нормированной форме, имеем:

$$
v_{i L} \in M_{C 0} I_{t}, \quad v_{i R} \in M_{Q} I_{t} ;
$$

рабочим множеством, регулирующим значения $v_{i}=i / l[i]$, здесь выбирается $M_{C 0} I_{t}$. Нормированные же величины $v_{u L}, v_{u R}$ принадлежат множеству $M_{Q} I_{t}$, к которому следует отнести и величину $v_{u}=u / 1[u]$.

Кроме параметров типа $v_{\text {п }}\left(\Pi_{a}\right)$ в выражения критериев подобия могут входить также обратные параметры, вида $v_{\text {побр }}=1 / v_{\text {п }}, \Pi_{\text {аобр }}=1 / \Pi_{a}$, причем 


$$
\begin{gathered}
v_{u C}=v_{1 / C} v_{q C}=v_{1 / C} \int_{I_{t}} v_{i c} d t, \quad v_{l / c}=\left(1 / C_{a}\right) /\left(\left[1 / C_{a}\right]\right) ; v_{i R}=v_{G} v_{u R}, \quad v_{G}=G /(I[G]), G=1 / R ; \\
v_{i L}=v_{l / L} v_{\psi L}=v_{1 / L} \int_{I_{t}} v_{u L} d t, \quad v_{l / L}=\left(1 / L_{a}\right) /\left(I\left[1 / L_{a}\right]\right) .
\end{gathered}
$$

Все реализации параметров типа $v_{\text {побр }}\left(\Pi_{\text {аобр }}\right)$ принадлежат тем же множествам, которые реализуют тип параметров $v_{\text {п }}\left(\Pi_{a}\right)$. В частности, для случая $v_{\text {п }}=$ const $\left(v_{\text {п }} \in \bar{R}\right)$ имеем $v_{\text {побр }} \in \bar{R}$, а для варианта $v_{\text {п }}=v_{\text {п }}(t)=v a r, \quad v_{\text {п }}(t) \in M_{\text {пп }} I_{t}$ выполняется условие $v_{\text {побр }}(t) \in M_{\text {vп }} I_{t}$.

Схемы, которые отражают взаимосвязи множеств, реализующих параметры типа $v_{\text {побр }}$, величин типа $v_{s}$ и критериев подобия, строятся согласно тому же подходу, что и схемы взаимосвязей критериев подобия с участием множества, обеспечивающего реализацию первоначально рассмотренных параметров-аналогов типа $v_{\text {п }}$. При рассмотрении величин, связанных с выработкой модельных аналогий электрической, магнитной, механической, гидравлической (пневматической) цепей [2], обращает на себя внимание тот факт, что произведение величинаналогов напряжения и тока каждый раз представляет собой мощность $p$, развиваемую данной цепью. Поэтому при построении операционных моделей-аналогов физических цепей возможно также использование энергетического критерия подобия

$$
p=u i ; v_{p}=v_{u} v_{i} ; v_{p}=p /(I[p]) \in M_{Q} I_{t} ; p \in M_{p} I_{t}=\left\{l[p] v_{Q} ; v_{Q} \in M_{Q} I_{t}\right\} .
$$

Итак, результатами отраженного в настоящей работе исследования следует считать, вопервых, получение совокупности критериев подобия физических цепей, специально ориентированных на базовые типы внутрицепных параметрических элементов, и, во-вторых, определение связей между критериями подобия и множествами различных реализаций модельных величин и параметров.

\section{БИБЛИОГРАФИЧЕСКИЙ СПИСОК}

1. Островский Л.А. Основы общей теории электроизмерительных устройств. Л.: Энергия, 1971. 544c.

2. Зарипов М.Ф., Никонов А.И., Петрова И.Ю. Элементы теории информационных моделей преобразователей с распределенными параметрами. Уфа: Башкир.филиал АН СССР, 1983.156с.

3. Бессонов Л.А. Теоретические основы электротехники: Учебник для вузов. М.: Высш. шк., 1964. 751с.

4. Треногин B.A. Функциональный анализ: Учеб. пособие для вузов. М.: Наука, 1980. 496с.

5. Рудин У. Основы математического анализа / Пер.с англ. М.: Мир, 1966. 319с.

6. Хатсон В., Пим Дж. Приложения функционального анализа и теории операторов: Пер. с англ. М.: Мир, $1983.432 \mathrm{c}$ 